\title{
Robustness of the Tuning of Fly Visual Interneurons to Rotatory Optic Flow
}

\author{
Katja Karmeier, ${ }^{1}$ Holger G. Krapp, ${ }^{2}$ and Martin Egelhaaf ${ }^{1}$ \\ ${ }^{1}$ Bielefeld University, Lehrstuhl für Neurobiologie, 33501 Bielefeld, Germany; and ${ }^{2}$ Department of Zoology, University of Cambridge, \\ Cambridge CB2 3EJ, United Kingdom
}

Submitted 11 March 2003; accepted in final form 30 April 2003

\begin{abstract}
Karmeier, Katja, Holger G. Krapp, and Martin Egelhaaf. Robustness of the tuning of fly visual interneurons to rotatory optic flow. J Neurophysiol 90: 1626-1634, 2003. First published May 7, 2003; 10.1152/jn.00234.2003. The sophisticated receptive field organization of motion-sensitive tangential cells in the visual system of the blowfly Calliphora vicina matches the structure of particular optic flow fields. Hypotheses on the tuning of particular tangential cells to rotatory self-motion are based on local motion measurements. So far, tangential cells have never been tested with global optic flow stimuli. Therefore we measured the responses of an identifiable neuron, the V1 tangential cell, to wide-field motion stimuli mimicking optic flow fields similar to those the fly encounters during particular self-motions. The stimuli were generated by a "planetarium-projector," casting a pattern of moving light dots on a large spherical projection screen. We determined the tuning curves of the V1-cell to optic flow fields as induced by the animal during 1 ) rotation about horizontally aligned body axes, 2) upward/downward translation, and 3) a combination of both components. We found that the V1-cell does not respond as specifically to self-rotations, as had been concluded from its receptive field organization. The neuron responds strongly to upward translation and its tuning to rotations is much coarser than expected. The discrepancies between the responses to global optic flow and the predictions based on the receptive field organization are likely due to nonlinear integration properties of tangential neurons. Response parameters like orientation, shape, and width of the tuning curve are largely unaffected by changes in rotation velocity or a superposition of rotational and translational optic flow.
\end{abstract}

\section{N T R O D U C T I O N}

To perform adequate motor actions, a mobile observer requires information about both its self-motion and the threedimensional layout of the environment. Visually orientating animals, including humans, obtain such information by exploiting optic flow induced during self-motion (Gibson 1979; Koenderink and van Doorn 1987; Warren et al. 2001). The global structure of optic flow depends on the performed self-motion: rotation about the longitudinal body axis, for instance, causes optic flow that is directed downward in the lateral visual field of one eye but upward in the lateral field of the other (Fig. 1A). During upward translation, the optic flow in the whole visual field is directed downward (Fig. $1 B$ ). While the global structure of rotatory and translatory flow fields can be distinguished quite easily, there may be ambiguities at a local scale (cf. Fig. $1, A$ and $B$, shaded squares in left part of middle panels). Obtaining unambiguous information requires neuronal mech-

Address for reprint requests: K. Karmeier, Bielefeld University, Lehrstuhl für Neurobiologie, Postfach 100131, D-33501 Bielefeld, Germany (E-mail: kkarmeier@uni-bielefeld.de). anisms that combine local motion information from large parts of the visual field in an appropriate way.

In vertebrates and invertebrates, neurons responding to optic flow have been found to have large receptive fields (e.g., Ibbotson 1991; Leonard et al. 1988; Wicklein and Varju 1999; Wylie and Frost 1999b). In the fly visual system, about 50-60 individually identifiable tangential cells integrate output signals of many local motion sensitive elements from large parts of the receptive field (reviews: Borst and Haag 2002; Egelhaaf et al. 2002; Hausen and Egelhaaf 1989). The local preferred directions within the receptive field of each tangential cell are not homogeneously oriented but match the orientation of velocity vectors in a particular optic flow field (review: Krapp 2000). The local preferred directions within the receptive field of tangential cells belonging to the vertical system (VS-cells; Hengstenberg et al. 1982), for instance, match the orientation of velocity vectors in optic flow fields induced during selfrotations of the animal. Their receptive field organization was concluded to be adapted to sense self-rotations about horizontally aligned body axes (Krapp and Hengstenberg 1996). This is also true for the tangential cell investigated in this study, the V1-cell, although it does not receive input directly from local motion sensitive elements, but from three VS-cells (Kurtz et al. 2001; Warzecha et al. 2003). Thus its receptive field structure resembles, as the receptive fields of VS-cells, the structure of a rotatory rather than a translatory flow field (Fig. 1C). Accordingly, the V1-cell can be expected to be tuned to a rotation of the fly about a horizontally aligned axis. These hypotheses on the tuning of tangential cells to particular types of selfmotion are based on local motion measurements. Verification of the predictions based on the local responses necessitates a stimulus covering large parts of the receptive field and approximating the local structures of an optic flow field.

In most studies investigating the response characteristics of optic flow neurons, simplifications have been made concerning the extent and/or the fine structure of optic flow stimuli. Relatively simple grating or dot patterns have been generated on displays, by rotating drums or by banks of light emitting diodes (e.g., fly: Hausen 1982; bee: Ibbotson et al. 1991; dragonfly: Olberg 1981; locust: Baader 1991; crab: Johnson et al. 2002; cat: Sherk et al. 1995; monkey: Duffy and Wurtz 1991). In this study, we used a "planetarium-projector" generating a pattern of moving light dots that more closely approximates realistic global optic flow. Although similar devices have been used in

\footnotetext{
The costs of publication of this article were defrayed in part by the payment of page charges. The article must therefore be hereby marked "advertisement" in accordance with 18 U.S.C. Section 1734 solely to indicate this fact.
} 
A Rotation

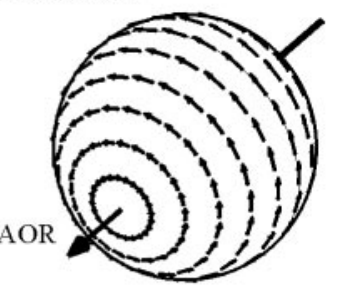

B Translation

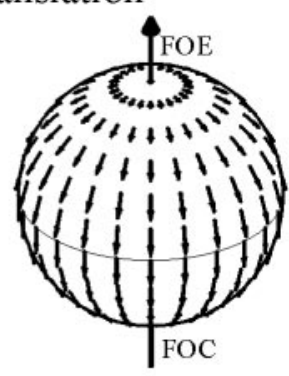

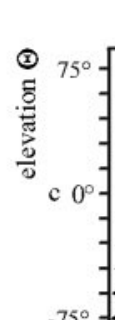

d
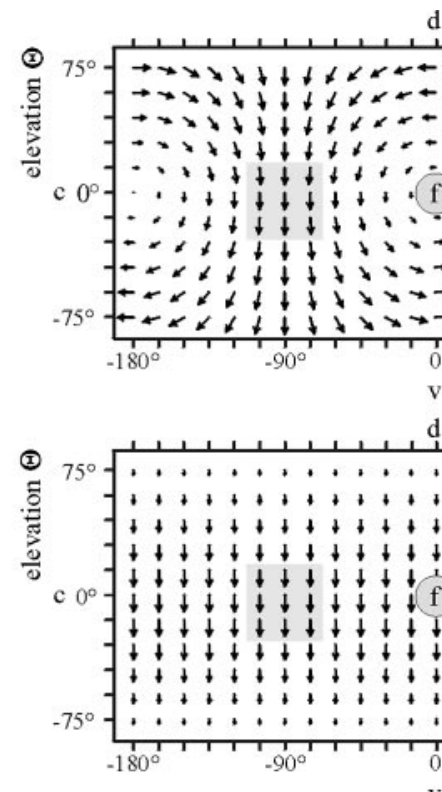

..........

$180^{\circ}$

$-90^{\circ}$

$+\dot{1}$

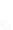

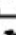

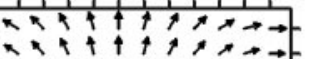

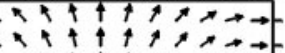

$+i+i+i, \overrightarrow{0}$

$+i+1+t, i ;$

$+t+\uparrow t+1$

$+t+\uparrow+1+i$

$\rightarrow+4+1$ is

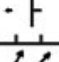

C Response field V1

\section{$d$}

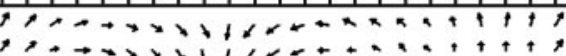

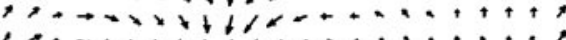

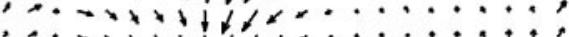

$\cdots+\cdots+1+1 / 1 \ldots \ldots \ldots+\cdots$

$\cdots \cdots+1+1, b+\cdots, \ldots$

$\cdots+1+1+b_{1} \cdots \cdots \cdots ;$

$\cdots \cdots+1+1\}+1+\cdots \cdots \cdot \cdots+\cdots$

$\cdots \cdots+1+1+\cdots \cdots+\cdots, \cdots$

$\cdots+\cdots+1+1+\cdots+\cdots+\cdots$

$\ldots \ldots+\ldots, \ldots, \ldots, \ldots$

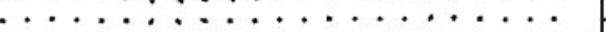

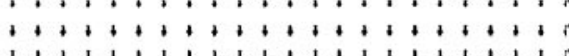

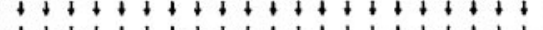

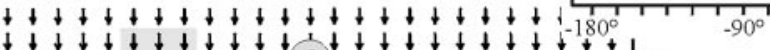

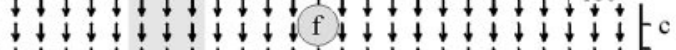

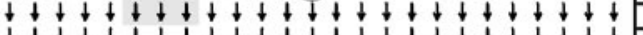

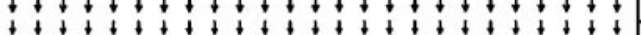

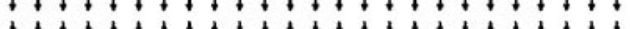

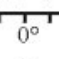

$\mathrm{v}$

azimuth $\Psi$

FIG. 1. Self-motion induced optic flow and response field of the V1-cell. Optic flow induced during a rotation about a horizontal $(A)$ or a upward translation along the vertical body axis $(B)$. Flow fields are illustrated in spherical coordinates of the visual field (left) and their projections onto Mercator maps (right). Positions in the Mercator map are specified by the angles of azimuth $(\Psi)$ and elevation $(\Theta) ; \Psi<0$ denotes left, $\Psi>0$ the right visual hemisphere ( $\mathrm{f}=$ frontal, $\mathrm{c}=$ caudal, $\mathrm{d}=$ dorsal, $\mathrm{v}=$ ventral). Each single arrow indicates the direction and velocity of the respective local image shift. Note that, due to the Mercator projection, the dorsal and ventral parts of the spherical visual field are highly overemphasized in the map. Rotation induces optic flow where local image shifts are aligned along parallel circles centered with the axis of rotation (AOR; $A$, left). In contrast, local image shifts induced by translation are aligned along great circles connecting the focus of expansion (FOE) with the focus of contraction (FOC; $B$, left). C: V1 response field. Binocular response field reconstructed from experiments in 4 animals. Orientation of each single arrow indicates the local preferred direction and its length gives the local motion sensitivity normalized to the maximum response. The V1 response field comprises almost the entire visual field. Maximum sensitivity is found in the azimuth range of $-15^{\circ}$ to $-45^{\circ}$ around the eye's equator. The local preferred directions of V1 continuously change from vertical downward in the frontolateral, to horizontal in the dorsolateral, to oblique vertical upward in the dorsocaudal visual field. The organization of the receptive field is more similar to the structure of a rotatory than to that of a translatory flow field, thus indicating that the V1-cell should be more sensitive to a self-rotation.

electrophysiological experiments on rabbits and pigeons (Simpson et al. 1988; Wylie et al. 1998), for technical reasons it was not possible, so far, to superimpose rotation and translation induced optic flow. Our planetarium projector overcomes these limitations in that it allows us to combine translatory and rotatory optic flow.

With this stimulus device we measured for the first time the activities of a particular fly tangential cell, the V1-cell, to realistic wide-field optic flow induced during different selfrotations and self-translations of the animal and a combination of both. We tested the specificity of the V1-cell to its preferred self-motion and how well the tuning of the cell can be predicted on the basis of its local response properties.

\section{METHODS}

\section{Preparation and electrophysiology}

All experiments were performed on 1- to 3-day-old female blowflies (Calliphora vicina) bred in our laboratory culture. To avoid inbreeding, the culture is refreshed several times a year with animals that were caught in the wild. Before dissection, the animals were briefly anesthetized with $\mathrm{CO}_{2}$. Legs and wings were removed, and the animal was fixed to a holder. Wounds were closed with wax to prevent the animal from desiccation. The head was aligned by adjusting it according to the symmetrical deep pseudopupil (Franceschini 1975) in the frontal region of both eyes. To access the lobula plate, the head capsule was opened from behind and fat tissue, air sacs, and tracheae were removed. By adding saline solution (for chemical composition, see Karmeier et al. 2001), the nervous tissue was kept moist. All experiments were performed at a temperature between 24 and $27^{\circ} \mathrm{C}$. All experiments were done in compliance with institutional guidelines.

The V1-cell can be identified unambiguously on the basis of its sensitivity to vertical downward motion in the visual field contralateral to the neuron's output ramifications in the lobula plate (Hausen 1976; Krapp and Hengstenberg 1997). From the output ramifications, we recorded extracellularly the spike activity of V1 using tungsten electrodes. The electrode tips were sharpened electrolytically and insulated with varnish resulting in impedances between 2 and $8 \mathrm{M} \Omega$. A tip-broken glass capillary was used as ground electrode and to supply the brain with saline solution. Recorded signals were processed by standard electrophysiological equipment and were sampled into a $\mathrm{PC}$ at a rate of $10 \mathrm{kHz}$ using a Data Translation board (DT 3001). Programs for data acquisition were written in HP VEE (HewlettPackard). All data were evaluated off-line using MATLAB (The Mathworks, Natick, MA).

\section{Visual stimulation}

Optic flow stimuli were generated by a planetarium projector (Fig. $2 A$ ) comparable to those used in electrophysiological experiments on rabbits and pigeons (Simpson et al. 1988; Wylie and Frost 1993). Our projector consisted of a small, hollow metal globe (120 $\mathrm{mm}$ diam), the surface of which was drilled with numerous small holes (30 within a solid angle of $90^{\circ}$ ). A small halogen light source was positioned in its center, casting a field of dots on the wall of a large, spherical 
A Setup

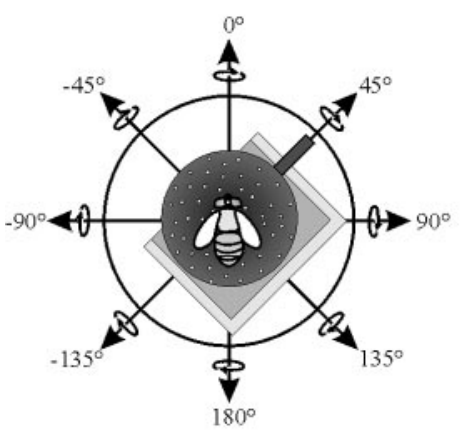

B Average response

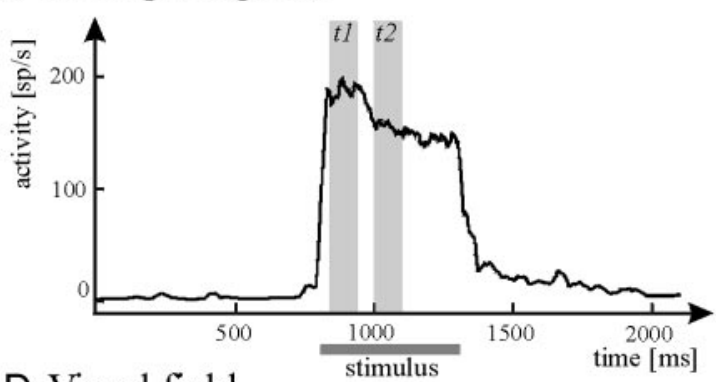

D Visual field

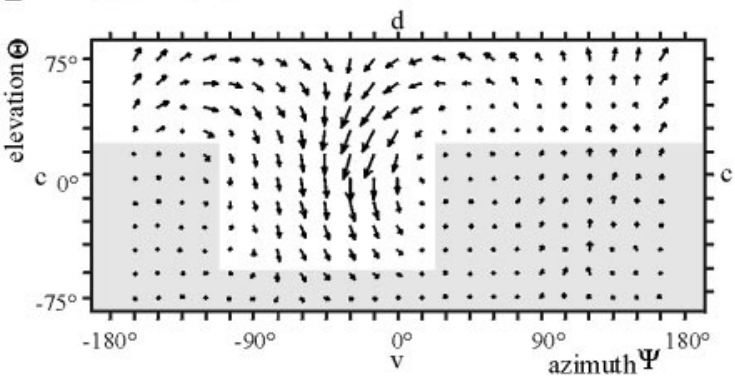

C Tuning curve

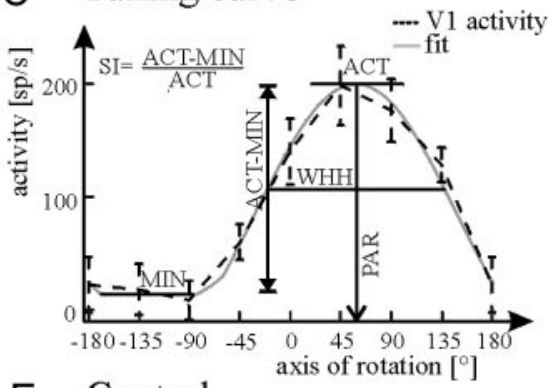

E Control

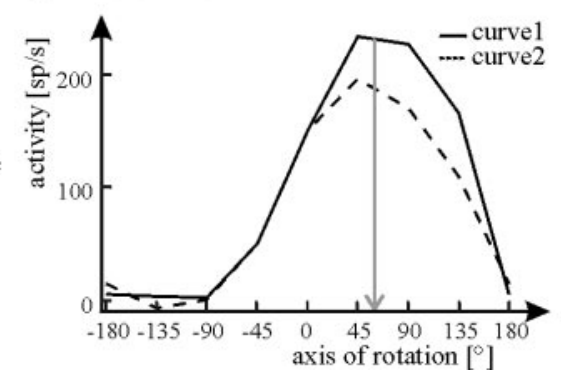

FIG. 2. A: experimental set-up. The light source positioned in the center of a small metal globe $(120 \mathrm{~mm}$ diam $)$ casts through small holes in the globe a field of light dots on a large spherical projection screen (1.2 $\mathrm{m}$ diam; data not shown). The fly is positioned in the center of the projection screen, above the metal globe. Rotation of the metal globe results in rotatory optic flow and translation of the light source within the metal globe results in translatory optic flow being projected to the screen. Tuning curves are obtained for rotations about horizontally aligned body-axes spaced $45^{\circ}$. The figure shows the coordinate system used in subsequent figures. Rotation of the metal globe about the $0^{\circ}$ axis corresponds to a self-rotation of the animal about the longitudinal body-axis to the right, rotation of metal globe about the $180^{\circ}$ axis to rotation of the animal about the same axis to the left. $B$ : activity of the V1-cell to a rotation about the $45^{\circ}$ axis at $390^{\circ} / \mathrm{s}$ plotted over time. Activity is smoothed with a sliding window of $40 \mathrm{~ms}$ width. The resting activity of the neuron is set to 0 . Horizontal bar below the $x$ axis indicates the stimulus period. To determine tuning curves, the average activity to a rotation about each axis was determined in 2100 -ms time windows, $t 1$ and $t 2$, starting 40 and $200 \mathrm{~ms}$ after motion onset, respectively (vertical bars). $C$ : average activity obtained within $t 1$ is plotted over the axis of rotation. To determine the parameters of interest, a cosine function was fitted to the data (see METHODS). PAR, azimuth of preferred axis of rotation, obtained from cosine fit; ACT, maximum activity obtained from cosine fit; WHH, width at half-maximum height; SI, sensitivity index, MIN, minimum of the fit. In this and the subsequent figures the preferred axis of rotation is indicated by an arrow pointing to the $x$ axis. $D$ : due to the construction of the projector, the metal globe is partly screened at 3 of 4 sides (cf. A) leading to a restricted projection of the optic flow fields. The gray area within the V1 response field indicates the area not visible to the fly at a rotation axis of $45^{\circ}$ and $-135^{\circ}$, respectively. Note that a change of the orientation of the projector results in a change of the area visible to the animal. $E$ : to test for the effect of this screening, we measured 2 tuning curves, each based on 5 different orientations of the rotation axis of the projector (see METHODS).

projection screen (1.2 $\mathrm{m}$ diam). The projected light dots were patches of nonhomogenous light with a mean luminance of $30 \mathrm{~cd} / \mathrm{m}^{2}$. Their size ranged from $1.9^{\circ} \times 3.8^{\circ}$ to $3.8^{\circ} \times 3.8^{\circ}$ as seen from the animal. Step motors (SIG Positec BERGERLAHR, Phytron) rotated the metal globe about three axes of a Cartesian coordinate systems. One horizontal axis allowed for continuous rotation. About the other two axes, periodic rotatory movements could be performed in the range of about $\pm 15^{\circ}$. Rotations of the metal globe resulted in coherently moving light patterns on the projection screen, mimicking rotatory optic flow. At the same time the light source inside the metal globe could be moved along three axes (up/down, right/left, forward/backward). The wide-field stimulus covered the entire dorsal-equatorial visual field except a small dorsal area of $22.6^{\circ}$ in diameter and a caudal area directly behind the animal. Relative to the eye equator the caudal area amounted to $\pm 30^{\circ}$ and $\pm 35^{\circ}$ in its horizontal and vertical extend, respectively. The fly's field of view was extended ventrally down to an elevation of $-45^{\circ}$ and for a range of approximately $120^{\circ}$ along the azimuth (extended visible section; Fig. $2 D$ ). The area in which no visual motion was presented is linked to the horizontal rotation axis of the device and therefore changed its azimuth as different axes of rotation are tested. The maximum speed with which we could move the light source within the metal globe mimicked the animal translating at $0.35 \mathrm{~m} / \mathrm{s}$ in an environment where the distance between its eyes and any visual contrasts amounted to $1 \mathrm{~m}$. This corresponded to an angular velocity of $39 \%$ at the flow field equator. Rotational velocities ranged from $39^{\circ} / \mathrm{s}$ to $1223 \%$ s.

\section{Determining tuning curves}

The fly was positioned in the center of the projection sphere, slightly above the metal globe as indicated in Fig. $2 A$. Rotatory tuning curves were obtained by recording the neuron's activity induced during simulated rotations about horizontal axes spaced at $45^{\circ}$. The curved arrows in Fig. $2 A$ indicate the direction of simulated selfrotation of the fly. Flow fields induced by self-rotations in opposite directions (e.g., $0^{\circ}$ and $180^{\circ}$ ) were generated by rotations of the metal globe in clockwise and counterclockwise direction, respectively. Therefore to determine the tuning curves, we effectively rotated the metal globe around five different axes of the planetarium projector. The stimulus consisted of $500 \mathrm{~ms}$ of motion in one direction and a stationary phase of $5 \mathrm{~s}$, followed by $500 \mathrm{~ms}$ of motion in the opposite direction. Average responses for each animal were obtained from two motion sweeps in either direction. We defined the responses to motion by subtracting the mean spike rate recorded within $500 \mathrm{~ms}$ before stimulus onset, from the mean spike rate measured within a $100-\mathrm{ms}$ time window during motion. To account for the time dependence of the responses, we determined the activity for two time windows (Fig. $2 B, t 1$ and $t 2$ ), starting 40 and $200 \mathrm{~ms}$ after stimulus onset, respec- 
tively. The starting time of the second window was constrained by the limited duration of the stimulus for combined motion. The average responses obtained during $t 1$ are plotted as a function of the orientation of the rotation axis (Fig. 2C). To facilitate the quantitative analysis of the experimental data, we applied a least square algorithm to fit a truncated cosine function to each of the measured tuning curves. The fits were based on four parameters: i.e., phaseshift, amplitude, cut-off, and offset. From the fits we obtained three parameters, which characterize the tuning of the neuron: 1) the "preferred axis of rotation" corresponds to the azimuth resulting in the maximum in the fitted tuning curve; 2) as the maximal neuronal response we consider the maximum of the fit; and 3) we define the width of the tuning curve by its width at half-maximum height of the fit. We additionally calculated a sensitivity index (for definition, see Fig. 2C).

\section{Control experiments}

As a result of the design of the stimulus device, the projected optic flow field was restricted in size (cf. Figs. $2 A$ and $2 D$ ). Additionally, a change of the projector orientation results in a change of the position of the extended visible section. To test whether these restrictions affect the tuning curves, we measured two tuning curves each of which was based on five different orientations of the projector. For the first curve (curve 1 in Fig. 2E), the projector was oriented in a way so that the extended visible section of the flow field was projected into the most sensitive part of the neuron's receptive field (extended visible section centered at $-135^{\circ},-90^{\circ},-45^{\circ}, 0^{\circ}$, and $45^{\circ}$ ). The second curve (curve 2 in Fig. $2 E$ ) was obtained by applying the opposite orientations (extended visible section centered at $45^{\circ}, 90^{\circ}$, $135^{\circ}, 180^{\circ}$, and $-135^{\circ}$ ). Although the resulting tuning curves show differences in the respective activity, both, the preferred axis and the width are similar. The positions of the projector used to derive the first curve were used in subsequent experiments.

\section{Calculation of tuning curves from local responses}

The local preferred directions and local motion sensitivities were determined by using a stimulus procedure introduced by Krapp and Hengstenberg (1997). In short, a small black dot $\left(7.6^{\circ}\right.$ diam) was moved at constant speed on a circular path $\left(10.4^{\circ}\right.$ diam $)$, thus covering only a small part of the visual field. When the instantaneous direction of dot motion coincided with the local preferred direction, the response was maximal. The local motion sensitivity is given by the difference between the average response to motion in an interval of $\pm 45^{\circ}$ relative to local preferred direction and the average response to motion in an interval of equal size relative to the null direction; i.e., $180^{\circ}$ apart from the local preferred direction. To quantify the similarity between the receptive field and the optic flow field, both fields were normalized with respect to the maximum local motion sensitivity or the maximum local velocity vector, respectively. The value obtained by projecting the optic flow field into the response field of the neuron indicates the similarity between both structures. To account for the restricted size of the stimulus, local scalar products of only those positions in the visual field were included in the integration, which were visible to the fly on stimulation with the planetarium projector. Distortions due to the Mercator-projection (see legend of Fig. 1) were compensated for by appropriate weighting of the local scalar products. Contributions from the left and right visual field were summed separately. To account for the rectification caused by spike generation, the contributions were set to zero if they became negative. Contributions from either side were added. Tuning curve parameters and the sensitivity index were derived directly from the calculated curves.

\section{RES U L T S}

To examine the tuning of the fly tangential cell V1 to rotatory self-motion, we measured its activities to flow fields mimicking rotations of the animal about different horizontally aligned body axes. The preferred axis of rotation, the width at half modulation height of the tuning curve, and the sensitivity index were derived from fits to the experimentally obtained tuning curves. These parameters were used to quantify the tuning while the angular velocity was varied or translatory optic flow was added to the rotatory flow. Unless otherwise stated the data represent the mean \pm SD obtained from five animals. The measured tuning curve parameters were compared with those obtained from the tuning curves calculated from local measurements.

\section{Tuning to pure rotation}

The dependence of the $\mathrm{V} 1$ response on the axis of rotation was tested for a wide range of velocities $(39 \%$, $123 \%$ s, 390\%, and $1223 \%$ s). Within both an early and a later time window of the response, the resulting tuning curves coincide largely. They have a peak in the range between approximately $55^{\circ}$ and $65^{\circ}$ in azimuth (Fig. 3, response $t 1$ and $t 2$; Table 1 ). On both sides of the peak, the sensitivity decreases. The width of the tuning curves at half-maximal amplitude is relatively broad (Table 1). Although the rotation velocity shows only little effect on the overall shape of the tuning curves, the response amplitudes in both time windows depend on velocity as has been described for other tangential cells before (Egelhaaf and Borst 1989; Hausen 1982; Maddess and Laughlin 1985).

To compare the tuning curves obtained with wide-field optic flow with the prediction based on the local preferred directions and local motion sensitivities within the receptive field of the V1-cell, the experimental data were fitted by a truncated cosine

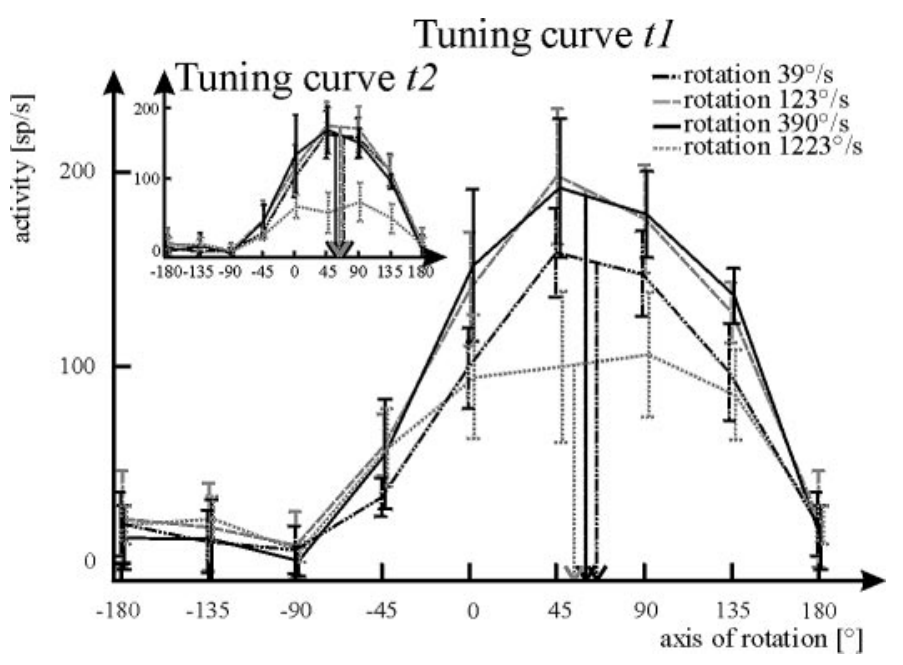

FIG. 3. Tuning curves measured with wide-field optic flow stimuli for 4 different rotation velocities. Experiments were done in 11 animals, each tested for 1-3 stimulus conditions. Mean activity and SD obtained from 5 (rotation velocity: $39 \%$ s, $123 \%$ s, and $390 \%$ s) or 4 animals (rotation velocity: $1223 \%$ s) are plotted over the respective axis of rotation. The resting spike activity is set to 0 . Arrows indicate the preferred axis for each tuning curve as obtained form the cosine fit. The V1-cell is broadly tuned to a rotation about an axis intermediate between the longitudinal $\left(0^{\circ}\right)$ and the transverse $\left(90^{\circ}\right)$ body axis. The mean preferred axis is at $60^{\circ}$ azimuth; the individual axes differ from the mean by maximally $5.25^{\circ}$ (rotation velocity: $39 \%$ s). Compared with the activity to a rotation about the preferred axis at $39 \%$ s, the activities to velocities of $123 \%$ and $390 \%$ s differ by an amount of 1.2 and for a velocity of $1223 \%$ by 0.7 . The largest difference in the width of the tuning curves amounts to $5.7 \%$ (rotation velocity: $1223 \%$ s) of the mean width for all conditions. The shape of the tuning curves obtained for the 2 time windows $t 1$ and $t 2$ (inset) are basically the same. 
TABLE 1. Tuning curve parameters and SI for all stimulus combinations

Velocities for Rotation $(r)$ and Lift-Translation $(t)$

\begin{tabular}{|c|c|c|c|c|c|c|c|c|c|c|c|c|}
\hline & \multicolumn{3}{|c|}{$\begin{array}{l}\text { Motion Components } \\
\left(r=39^{\circ} / \mathrm{s} ; t=39^{\circ} / \mathrm{s}\right)\end{array}$} & \multicolumn{3}{|c|}{$\begin{array}{l}\text { Motion Components } \\
(r=123 \% \mathrm{~s} ; t=39 \% \mathrm{~s})\end{array}$} & \multicolumn{3}{|c|}{$\begin{array}{l}\text { Motion Components } \\
\left(r=390 \% \text { s } ; t=39^{\circ} / \mathrm{s}\right)\end{array}$} & \multicolumn{3}{|c|}{$\begin{array}{c}\text { Motion Components } \\
\left(r=1223^{\circ} / \mathrm{s} ; t=39^{\circ} \% \mathrm{~s}\right)\end{array}$} \\
\hline & $r$ & $r: t+$ & $r: t-$ & $r$ & $r: t+$ & $r: t-$ & $r$ & $r: t+$ & $r: t-$ & $r$ & $r: t+$ & $r: t-$ \\
\hline $\operatorname{PAR}\left({ }^{\circ}\right)$ & $65 \pm 7$ & $75 \pm 7$ & - & $59 \pm 7$ & $61 \pm 4$ & $61 \pm 9$ & $60 \pm 8$ & $56 \pm 8$ & $62 \pm 12$ & $55 \pm 9$ & $50 \pm 19$ & $55 \pm 21$ \\
\hline $\mathrm{ACT}(\mathrm{sp} / \mathrm{s})$ & $162 \pm 21$ & $180 \pm 29$ & - & $200 \pm 28$ & $185 \pm 29$ & $159 \pm 14$ & $205 \pm 28$ & $191 \pm 34$ & $145 \pm 18$ & $110 \pm 25$ & $96 \pm 13$ & $66 \pm 13$ \\
\hline WHH $\left(^{\circ}\right)$ & $153 \pm 10$ & $143 \pm 11$ & - & $158 \pm 5$ & $160 \pm 5$ & $138 \pm 7$ & $158 \pm 8$ & $166 \pm 7$ & $144 \pm 13$ & $164 \pm 10$ & $174 \pm 12$ & $167 \pm 17$ \\
\hline SI (relative units) & $.95 \pm .08$ & $.68 \pm .05$ & - & $.93 \pm .10$ & $.97 \pm .03$ & $.95 \pm .07$ & $.96 \pm .07$ & $1 \pm .02$ & $.99 \pm .07$ & $.86 \pm .08$ & $.94 \pm .05$ & $.98 \pm .17$ \\
\hline
\end{tabular}

Values are means \pm SD. Tuning curve parameters obtained from cosine fits to data measured with global motion stimuli. The translation velocity used in the experiments corresponds to an angular velocity of $39^{\circ} / \mathrm{s}$ in the flow field equator. Combination of rotation at $39^{\circ} / \mathrm{s}$ with negative translation leads to almost negligible responses. Therefore the tuning curve parameters for this motion combination have not been calculated. PAR, preferred axis of rotation; ACT, activity to rotation about preferred axis; WHH, width at half maximum height; SI, sensitivity index.

function (see Fig. 4 for the fit for a velocity of $123 \%$ ). The overall shape of the two curves is similar and the curves overlap greatly. However, three differences between the curves are obvious: 1 ) the preferred axis of rotation of the predicted and the measured curve differ by approximately $20^{\circ} ; 2$ ) the spike rate measured with the wide-field stimulus is never reduced to the resting level; and 3) the tuning curve measured with wide-field motion is broader than predicted. These findings are corroborated by experiments done on another tangential cell $(\mathrm{V} 2, n=1)$. The V2-cell receives retinotopic input and conveys motion information via a thin axon to the contralateral lobula plate where it forms output ramifications (Hausen 1984). V2 is most sensitive to upward motion in the lateral visual field and is suggested to sense roll rotations. The differences between the predicted and the measured tuning curves of

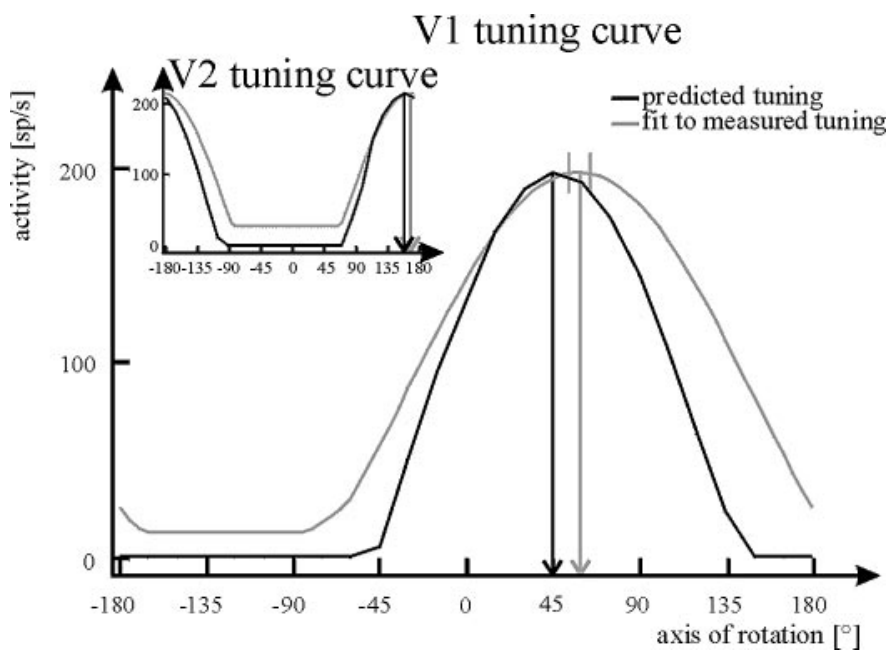

FIG. 4. Cosine fit to the tuning curve measured with wide-field rotatory optic flow at $123 \%$ s (resting spike activity set to 0 ) and tuning curve computed from local motion measurements (cf. METHODS). The calculated curve is scaled to the maximum of the fit; the predicted preferred axis is located at the maximum of the calculated tuning curve. The small vertical bars left and right to the maximum of the fit indicate the angular deviation of the preferred axis of rotation. Even if the overall shape of the 2 curves is similar, some differences are obvious: the preferred axis of the calculated and the measured curve differs by $20^{\circ}$ and the width of the calculated curve is smaller by $23 \%$ compared with the measured width. In addition, the neuron's spiking activity is not reduced to resting activity for a range of rotation axes $\left(-180^{\circ}\right.$ to $-45^{\circ}$ and $+150^{\circ}$ to $+180^{\circ}$ ) as predicted. Similar differences between measured tuning curve and tuning curve calculated from local motion measurements were found for another tangential cell, the V2-cell (inset). the V2-cell are basically the same as those described for the V1-cell (Fig. 4, inset).

\section{Response to pure lift translation}

The response field of the V1-cell resembles the structure of a flow field induced by a rotation about the neurons preferred axis. However, the vectors in an optic flow field induced during upward (positive) lift-translation match the local preferred directions in the V1-response field around an azimuth of $-30^{\circ}$ (Fig. 1B). Accordingly, some activity is predicted for a positive lift translation: it amounts to approximately $43 \%$ of that predicted for rotations about the preferred axis of the V1-cell (Fig. 5 ). The predicted specificity for rotatory optic flow is not found in the measured neuronal responses. The activities to a lifttranslation inducing maximal angular displacements of $39 \%$ amount to $92 \%$ of those evoked by a rotation about the preferred axis at $39 \%$ for both time windows.

\section{Tuning to rotation and superimposed lift translation}

So far, tuning to optic flow has only been characterized for purely rotatory or translatory optic flow. In behavioral situations, both motion components are usually superimposed. The structure of flow fields induced during a pure rotation or during combined rotational and translational movements may differ considerably. Because of the V1-cell's sensitivity to positive

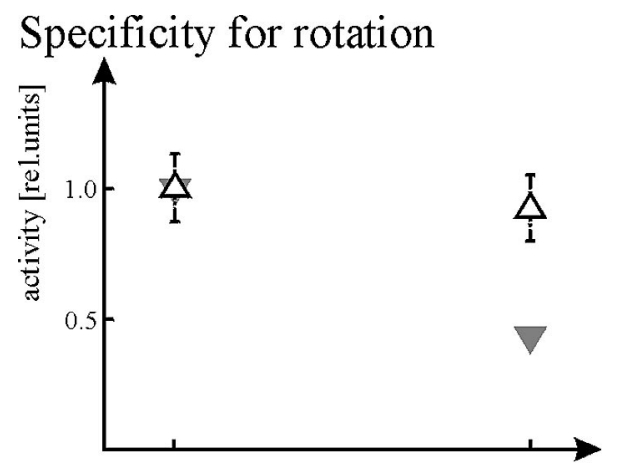

FIG. 5. Response to a rotation about the preferred axis of the V1-cell at $39 \%$ (left) and activity induced during positive lift-translation (right). In contrast to the measured activity (open black symbols), the model (filled gray symbols) predicts a weaker response to a pure lift translation than to a rotation about the neuron's preferred axis. Values are normalized to the response to rotation. 
lift translation, we chose to superimpose rotations with positive and negative lift-translations. Both stimuli induce maximum angular displacements of the pattern of $39^{\circ} \%$, corresponding to a rotation of $39 \%$ and a translation at $0.35 \mathrm{~m} / \mathrm{s}$. The superimposed lift-translation has the strongest effect on the rotation flow field in the equator of the visual field, where the downward motion component is doubled or canceled out, respectively (Fig. 6A). The predicted activities reflect this effect of the superimposed lift-translations: the activity increases when a positive lift and decreases when a negative lift is superimposed. The preferred axes of rotation, the width, and the overall shape of the predicted curves are hardly affected (Fig. 6B,top).

The effect of a superimposed lift-translation is much stronger for the measured activities; compared with the tuning curve obtained for pure rotation, the activity during rotations com- bined with a positive lift-translation is stronger for most axes of rotation, particularly for those axes where there are only small responses to a pure rotation (Fig. $6 \mathrm{~B}$, bottom). Despite these differences in the shape of the tuning curves, the preferred axes of rotation and the width of the curves are only slightly affected. The combination of rotations and a negative lifttranslation results in almost negligible responses for all axes of rotation. We were not able to compute the tuning curve parameters for this motion combination.

By superimposing a lift-translation of $0.35 \mathrm{~m} / \mathrm{s}$ on rotations of all tested angular velocities $\left(39^{\circ} / \mathrm{s}, 123 \% \mathrm{~s}, 390 \%\right.$, $1223 \%$ s), we changed the ratio of rotation:translation from $1: 1$ to $32: 1$. The shape of the tuning curves is relatively invariant when the rotation velocity is increased. The maximum response amplitude elicited by a rotation about the

\section{A Flow field structure}

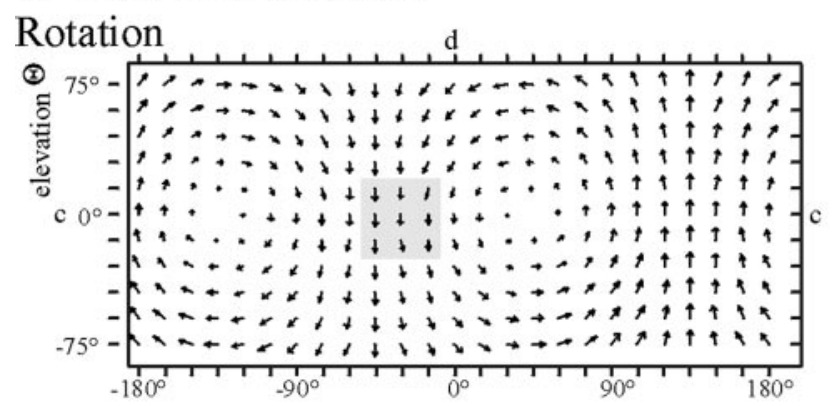

Rotation/Lift+

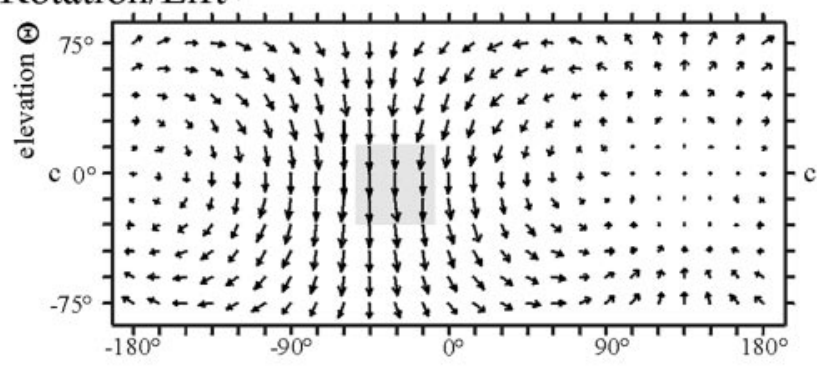

Rotation/Lift-

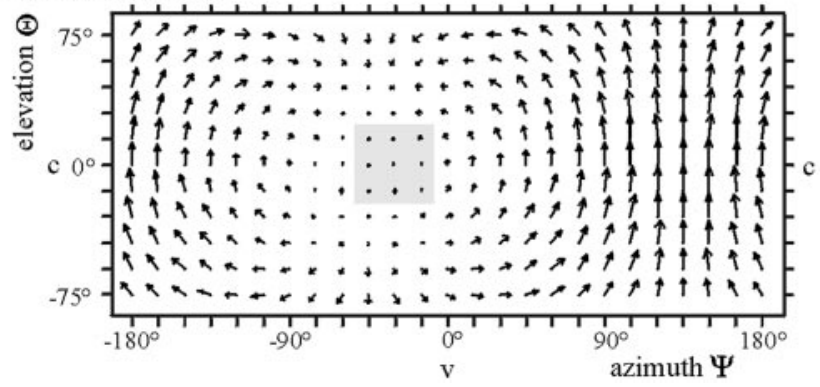

B Tuning curve
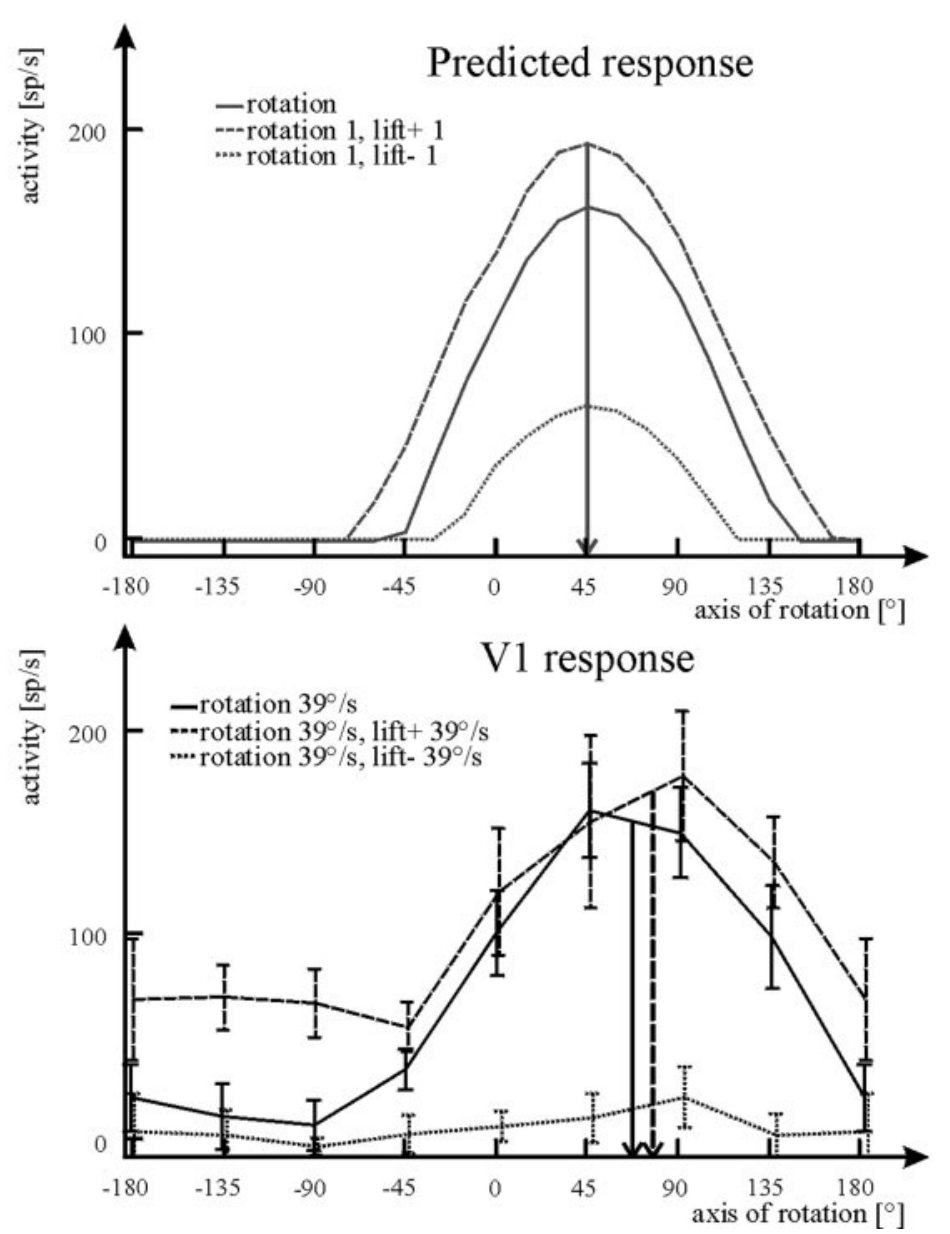

FIG. 6. Effect of lift-translation superimposed on rotatory flow. A: optic flow fields induced by a pure rotation about the measured preferred axis of the V1-cell $\left(\Psi=60^{\circ}\right)$. Rotatory movements were superimposed by positive and negative lifttranslations with the same angular velocity in the equator of the visual field $\left(\Theta=0^{\circ}\right)$. Shaded area in each flow field indicates the part of the receptive field where the V1-cell is most sensitive to downward motion. For the pure rotation (top), the local velocity vectors are pointing downward in this area. Superposition of positive lift (middle) leads to an increase, and superposition of negative lift (bottom) to a decrease of the downward motion component in this area. $B$ : tuning curves predicted from the local response properties of the V1-cell (top) and tuning curves obtained for pure rotation at $39 \%$ s and for rotations superimposed with lift-translations inducing angular displacements of $39 \%$ in the equator of the visual field (bottom). Data for pure rotation are the same as in Fig. 4. The overall shape of the predicted tuning curves is the same; the response amplitude and the width of the curves are affected by the superimposed lift-translation. For the measured curves, the preferred axis obtained from the tuning curve to rotation superimposed by positive lift-translation is shifted by $10^{\circ}$ from the preferred axis for pure rotation. Compared with the width of the tuning curves obtained with pure rotation, the width for the combined motion is smaller by $7 \%$. The combination of rotations and negative lift-translations results in almost negligible responses for all axes of rotation. 
cell's preferred axis depends on the rotation velocity but is hardly affected by a superimposed lift-translation (Fig. $7 D-$ $7 F$ ). The differences in the widths of the curves are relatively small compared with the overall tuning widths of the cell. Table 1 summarizes the tuning curve parameters for all stimulus conditions.

\section{I S C U S S I O N}

To gain information about self-motion from optic flow, tangential cells in the fly visual system have to pool the signals of many retinotopic inputs from extended parts of the visual field. Experiments using local motion stimuli indicate that the receptive field organization of some tangential cells is adapted to this task: the distribution of local preferred directions matches the distribution of velocity vectors in optic flow fields induced during certain selfmotions (Franz and Krapp 2000; Krapp 2000). Predictions based on the local motion measurements were tested here for the first time with realistic optic flow generated by a novel planetarium-like visual stimulator.

In the following, we will discuss 1 ) the differences between the measured and predicted specificity of the V1-cell to its preferred self-motion and possible reasons for the differences

\section{V1 response vs. calculated response}

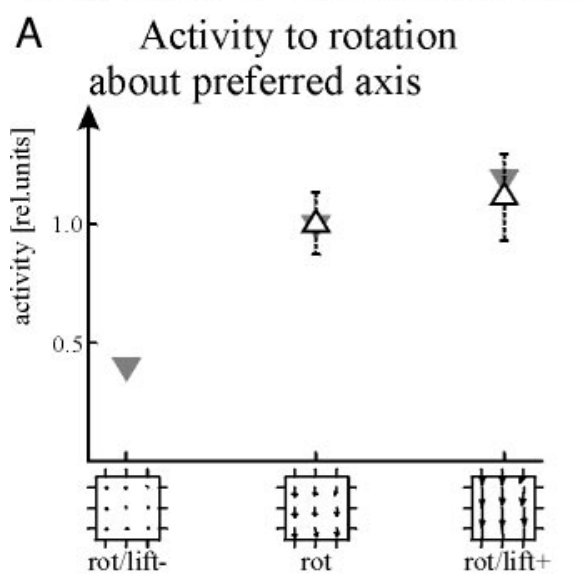

B Tuning width

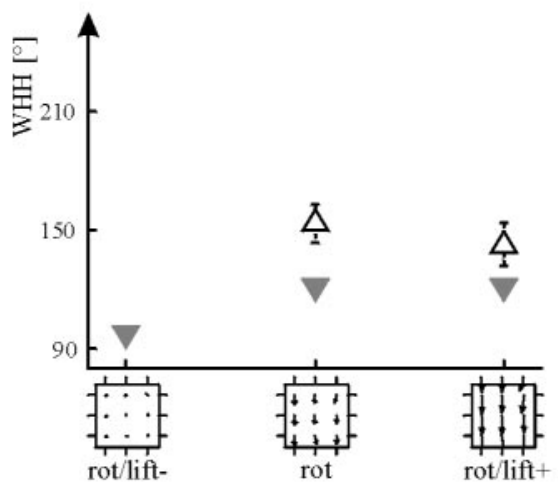

V1 response, all velocity ratios

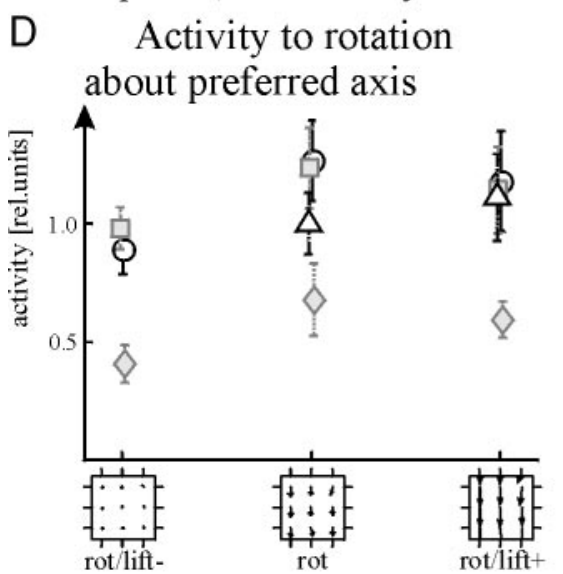

E Tuning width

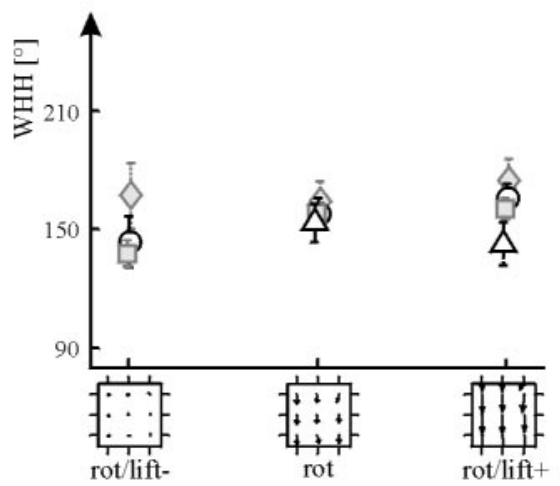

C Sensitivity index

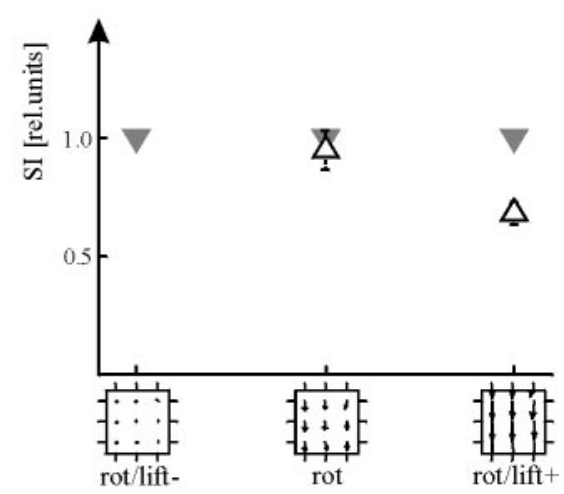

F Sensitivity index

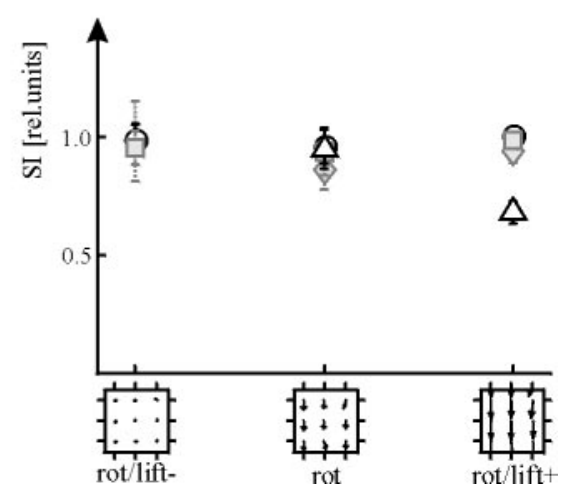

FIG. 7. Tuning curve parameters, mean and SD (smaller than symbol size if invisible). The symbols in the left of each panel indicate values for rotation superimposed with a negative lift-translation, symbols in the middle are values for pure rotation, and symbols in the right are values for rotation superimposed with positive lift-translation. Sketches below each panel schematize those sections of the respective optic flow stimulus, which affect the neuron's area of maximum sensitivity to downward motion (corresponding to the gray area in Fig. 6A). Top: parameters for the tuning curves measured with rotations of $39^{\circ} / \mathrm{s}$ (open symbols) and calculated curves (filled symbols) shown in Fig. $6 B$. Since combination of rotation and negative lift nearly leads to total inhibition of the measured response, tuning curve parameters were not determined for this motion combination. A: activity to rotation about preferred axis for measured tuning curve and for the predicted curve scaled to the measured data. Values are normalized to the activity to pure rotation. $B$ : width of measured and calculated tuning curves, $C$ : sensitivity index of measured and calculated curve. Bottom: parameters for tuning curves obtained from stimuli combining rotations and lift-translation at different velocity ratios $(\triangle$, rotation:translation: $39 \% \mathrm{~s}: 39 \%$ s; $, 123 \% \mathrm{~s}: 39 \%$ s; $0,390 \% \mathrm{~s}: 39 \%$ s; $, 1223 \% \mathrm{~s}: 39 \%$ s). Data for combinations with $1223 \%$ s rotation velocity obtained from 4 animals. $D$ : activity to rotation about preferred axis for all stimulus combinations, values normalized to response to pure rotation at $39^{\circ} / \mathrm{s}$. The activity is affected by the rotation velocity as described in Fig. 3. For all stimulus combinations, the response to rotation superimposed with a negative lift is smaller than to the pure rotation. E: superposition of a lift-translation on rotations with different velocities has no consistent effect on the width of the tuning curves. $F$ : sensitivity index for all motion combinations. Except the sensitivity index obtained from a motion combination of lift and rotation at $39 \%$, the width of the curves and sensitivity index are hardly affected by a change in the rotation velocity or superposition of a lift-translation. 
and 2) the tuning characteristics of the V1-cell in the context of encoding of self-motion from optic flow.

\section{Prediction of neuronal activities from local response properties}

The tuning curves predicted on the basis of local motion measurements do not agree in all aspects with the experimentally determined tuning curves. The predictions capture qualitatively the general shape of the experimental tuning curves (Fig. 4) and the overall effect of a superimposed lift translation (Fig. 6). The difference between the measured and predicted preferred axis of rotation is likely to be a consequence of the different set-ups in which the respective experiments were performed. However, there is a marked difference in the predicted and measured specificity to a rotation about the neuron's preferred axis: the measured tuning curve is broader (Fig. 4), the response to a rotation about the neuron's preferred axis and to a positive lifttranslation are in the same range (Fig. 5), and the impact of a superimposed lift translation is stronger than predicted (Figs. 6 and 7). These differences are most likely due to linear integration of local motion contributions in our model, which we tried to keep as simple as possible. Some nonlinearities known for fly tangential cells concern the computation of motion by local motion detectors and the spatial pooling of the motion detectors' outputs by the tangential cells. 1) The output of the local motion detectors is not proportional to velocity. It first increases with increasing velocity, reaches an optimum and then decreases again. It also depends on pattern properties like spatial wavelength and contrast (Egelhaaf and Borst 1993). Even the time course of a summed array of local motion detectors is proportional to pattern velocity only for relatively slow velocity changes (Egelhaaf and Borst 1993; Egelhaaf and Reichardt 1987). 2) The output of tangential cells is proportional to the number of activated local motion detectors only for small numbers. For greater numbers of inputs the neurons show a "saturation-like" characteristic (Borst et al. 1995; Hausen 1982; Hengstenberg 1982). This nonlinearity does not represent an output saturation at the level of TCs, but has been interpreted as a "gain control" mechanism because the level at which the response saturates depends on pattern velocity (Borst et al. 1995; Single et al. 1997; review: Egelhaaf and Warzecha 1999).

As a consequence of these nonlinearities, the V1-cell is likely to be activated maximally not only during a rotation about its preferred axis, but also if the structure of the optic flow field does not perfectly match the structure of the receptive field. In contrast, the activity predicted from the local response properties becomes maximal only if the optic flow field perfectly matches the structure of the receptive field. The predicted activities are smaller if the optic flow field does not perfectly match the distribution of local preferred directions. It is most likely that the nonlinear integration properties of the tangential cells result in the measured lack of specificity to rotatory self-motion, i.e., the broad tuning curves, the strong response to a positive lift-translation, and the stronger impact of superimposed translations.

In conclusion, the receptive field organization, as determined with local motion stimuli, is a good indicator of the preferred self-motion of a neuron but does not provide appropriate information about the neuron's specificity for its preferred selfmotion. A similarly small specificity to a neuron's preferred self-motion was found in different studies in invertebrates and vertebrates that analyze specificity by characterizing the cells with either, rotatory, and translatory optic flow stimuli (e.g., Duffy and Wurtz 1991; Hausen 1981; Horstmann et al. 2000; Ibbotson and Goodman 1990; Kern 1998; Kern et al. 2001; Tanaka et al. 1989). Studies where a stimulus device was chosen that could generate either translatory or rotatory optic flow could not provide appropriate information about the specificity to the preferred self-motion (e.g., Simpson et al. 1988; Tanaka and Saito 1989; Wylie and Frost 1999a).

\section{Tuning characteristics of the VI-cell in the context of encoding self-motion from optic flow}

To characterize the tuning of the V1-cell to its preferred self-motion, we have applied flow field combinations that are critical for the neuron rather than visual stimuli as encountered by the animal during everyday flight. The fly may hardly encounter a pure lift-translation and some of the generated rotation velocities are relatively slow compared with the velocities in normal flight situations (Hateren and Schilstra 1999; Schilstra and Hateren 1999). Furthermore, we simulated optic flow patterns within an environment assuming a uniform distance distribution. Nevertheless this systematic analysis helps to understand principles of encoding self-motion in the fly visual system. The findings from this study clearly show that a given activity level of the neuron does not provide unambiguous information about the stimulus. The magnitude of the cells response depends on rotation velocity (Fig. 3) and is in the same range for pure upward lift and for a rotation about the preferred axis (Fig. 5). Thus the same activity level in the V1-cell can be elicited, for instance, by a slow rotation about the preferred axis, by a fast rotation about a nonoptimal axis, or by a translational self-motion. Variability in the neuronal response even further limits the precision with which self-motion can be detected (e.g., Warzecha and Egelhaaf 1999).

These response ambiguities can be resolved, at least partly, if we take into account that self-motion information is conveyed by a population of tangential cells each responding best to a particular self-motion. A rotation about a particular axis induces a specific pattern of neuronal population activity. A change in rotation velocity or translatory motion superimposed on the rotation would have a similar effect on the activities of all neurons in the ensemble. The relative activities of the neurons are likely to be less affected. If the tuning width or the preferred axis of rotation of the neurons in the ensemble would depend on rotation velocity or on superimposed translation, it would be much more difficult to detect the axis of rotation from the pattern of population activity. Therefore an invariant tuning width and preferred axis of rotation seem to be advantageous for a robust neuronal representation of self-motion information.

We thank N. Böddeker, J. Grewe, S. Huston, R. Kern, and R. Kurtz for critically reading and discussing the manuscript. The helpful comments of two anonymous referees are gratefully acknowledged. We also thank the mechanical workshop of the Fakūtät für Biologie, Bielefeld University for constructing part of the stimulus equipment and D. Lohmann for programming the software used to control the "planetarium projector." 


\section{I S C L O S URES}

Parts of this work were supported by a grant from the Deutsche Forschūngs Gemeinschaft Graduate Program 518 (Strategies and Optimization of Behavior) to K. Karmeier.

\section{REFERENCES}

Baader A. Simulation of self-motion in tethered flying insects: an optical flow field for locusts. J Neurosci Methods 38: 193-199, 1991.

Borst A, Egelhaaf M, and Haag J. Mechanisms of dendritic integration underlying gain-control in fly motion-sensitive interneurons. J Comp Neurosci 2: 5-18, 1995

Borst A and Haag J. Neural networks in the cockpit of the fly. J Comp Physiol [A] 188: 419-437, 2002.

Duffy CJ and Wurtz RH. Sensitivity of MST neurons to optic flow stimuli. I. A continuum of response selectivity to large-field stimuli. J Neurophysiol 65: 1329-1345, 1991

Egelhaaf $\mathbf{M}$ and Borst $\mathbf{A}$. Transient and steady-state response properties of movement detectors. J Opt Soc Am A 6: 116-127, 1989.

Egelhaaf $\mathbf{M}$ and Borst A. Movement detection in arthropods. In: Visual Motion and Its Role in the Stabilization of Gaze, edited by Miles FA and Wallman J. New York: Elsevier. 1993, 53-77.

Egelhaaf M, Kern R, Krapp HG, Kretzberg J, Kurtz R, and Warzecha AK. Neural encoding of behaviourally relevant visual-motion information in the fly. Trends Neurosci 25: 96-102, 2002.

Egelhaaf $\mathbf{M}$ and Reichardt $\mathbf{W}$. Dynamic response properties of movement detectors: theoretical analysis and electrophysiological investigation in the visual system of the fly. Biol Cybern 56: 69-87, 1987.

Egelhaaf $M$ and Warzecha AK. Encoding of motion in real time by the fly visual system. Curr Opin Neurobiol 9: 454-460, 1999.

Franceschini N. Sampling of visual environment by the compound eye of the fly: fundamentals and applications. In: Photoreceptor Optics, edited by Snyder AW and Menzel R. New York: Springer, 1975, p. 98-125.

Franz MO and Krapp HG. Wide-field, motion-sensitive neurons and matched filters for optic flow fields. Biol Cybern 83: 185-197, 2000

Gibson JJ. The Ecological Approach to Visual Perception. London: Houghton Mifflin, 1979.

Haag $\mathbf{J}$ and Borst $\mathbf{A}$. Dendro-dendritic interactions between motion-sensitive large-field neurons in the fly. J Neurosci 22: 3227-3233, 2002.

Hateren JH and Schilstra C. Blowfly flight and optic flow. II. Head movements during flight. J Exp Biol 202: 1491-1500, 1999.

Hausen K. Functional characterization and anatomical identification of motion sensitive neurons in the lobula plate of the blowfly Calliphora erythrocephala. Z Naturforsch 31: 629-633, 1976.

Hausen K. Monocular and binocular computation of motion in the lobula plate of the fly. Verh Dtsch Zool Ges 74: 49-70, 1981.

Hausen K. Motion sensitive interneuron in the optomotor system of the fly. II. The horizontal cells: receptive field organization and response characteristics. Biol Cybern 46: 67-79, 1982.

Hausen K. The lobula-complex of the fly: structure, function and significance in visual behaviour. In: Photoreception and Vision in Invertebrates, edited by Ali MA. New York: Plenum Press, 1984, p. 523-559.

Hausen $\mathbf{K}$ and Egelhaaf M. Neural mechanisms of visual course control in insects. In: Facets of Vision, edited by Stavenga DG and Hardie RC. Berlin: Springer, 1989, p. 391-424.

Hengstenberg R. Common visual response properties of giant vertical cells in the lobula plate of the blowfly Calliphora. J Comp Physiol [A] 149: 179-193, 1982.

Hengstenberg R, Hausen K, and Hengstenberg $\mathbf{B}$. The number and structure of giant vertical cells (VS) in the lobula plate of the blowfly Calliphora erythrocephala. J Comp Physiol [A] 149: 163-177, 1982.

Horstmann W, Egelhaaf M, and Warzecha AK. Synaptic interactions increase optic flow specificity. Eur J Neurosci 12: 2157-2165, 2000.

Ibbotson MR. Wide-field motion-sensitive neurons tuned to horizontal movement in the honeybee, Apis mellifera. J Comp Physiol [A] 168: 91-102, 1991.

Ibbotson MR and Goodman LJ. Response characteristics of four wide-field motion-sensitive descending interneurons in Apis mellifera. J Exp Biol 148 255-279, 1990.

Ibbotson MR, Maddess T, and DuBois R. A system of insect neurons sensitive to horizontal and vertical image motion connects the medulla and midbrain. J Comp Physiol [A] 169: 355-367, 1991.
Johnson AP, Horseman BG, Macauley MW, and Barnes WJ. PC-based visual stimuli for behavioural and electrophysiological studies of optic flow field detection. J Neurosci Methods 114: 51-61, 2002.

Karmeier K, Tabor R, Egelhaaf M, and Krapp HG. Early visual experience and the receptive-field organization of optic flow processing interneurons in the fly motion pathway. Vis Neurosci 18: 1-8, 2001.

Kern R. Visual position stabilization in the hummingbird hawk moth, Macroglossum stellarum L. II. Electrophysiological analysis of neurons sensitive to wide-field image motion. J Comp Physiol [A] 182: 239-249, 1998.

Kern R, Lutterklas M, Petereit C, Lindemann JP, and Egelhaaf $M$. Neuronal processing of behaviourally generated optic flow: experiments and model simulations. Network 12: 351-369, 2001.

Koenderink JJ and van Doorn AJ. Facts on optic flow. Biol Cybern 56: 247-254, 1987.

Krapp HG. Neuronal matched filters for optic flow processing in flying insects. Int Rev Neurobiol 44: 93-120, 2000.

Krapp HG and Hengstenberg R. Estimation of self-motion by optic flow processing in single visual interneurons. Nature 384: 463-466, 1996.

Krapp HG and Hengstenberg R. A fast stimulus procedure to determine local receptive field properties of motion-sensitive visual interneurons. $\mathrm{Vi}$ sion Res 37: 225-234, 1997.

Kurtz R, Warzecha AK, and Egelhaaf M. Transfer of visual motion information via graded synapses operates linearly in the natural activity range. J Neurosci 21: 6957-6966, 2001.

Leonard CS, Simpson JI, and Graf W. Spatial organization of visual messages of the rabbit's cerebellar flocculus. I. Typology of inferior olive neurons of the dorsal cap of Kooy. J Neurophysiol 60: 2073-2090, 1988.

Maddess T and Laughlin SB. Adaptation of the motion-sensitive neuron H1 is generated locally and governed by contrast frequency. Proc $R$ Soc Lond B 225: 251-275, 1985.

Olberg RM. Object- and self-movement detectors in the ventral nerve cord of the dragonfly. J Comp Physiol 141: 327-334, 1981.

Schilstra C and Hateren JH. Blowfly flight and optic flow. I. Thorax kinematics and flight dynamics. J Exp Biol 202: 1481-1490, 1999.

Sherk H, Kim JN, and Mulligan K. Are the preferred directions of neurons in cat extrastriate cortex related to optic flow? Vis Neurosci 12: 887-894, 1995

Simpson JI, Leonard CS and Soodak RE. The accessory optic system of rabbit. II. Spatial organization of direction selectivity. J Neurophysiol 60: 2055-2072, 1988.

Single S, Haag J, and Borst A. Dendritic computation of direction selectivity and gain control in visual interneurons. J Neurosci 17: 6023-6030, 1997.

Tanaka K, Fukada Y, and Saito HA. Underlying mechanisms of the response specificity of expansion/contraction and rotation cells in the dorsal part of the medial superior temporal area of the macaque monkey. $\mathrm{J} \mathrm{Neu}$ rophysiol 62: 642-656, 1989

Tanaka K and Saito H. Analysis of motion of the visual field by direction, expansion/contraction, and rotation cells clustered in the dorsal part of the medial superior temporal area of the macaque monkey. J Neurophysiol 62: 626-641, 1989.

Warren WH Jr, Kay BA, Zosh WD, Duchon AP, and Sahuc S. Optic flow is used to control human walking. Nat Neurosci 4: 213-216, 2001.

Warzecha AK and Egelhaaf M. Variability in spike trains during constant and dynamic stimulation. Science 283: 1927-1930, 1999.

Warzecha AK, Kurtz R, and Egelhaaf M. Synaptic transfer of dynamic motion information between identified neurons in the visual system of the blowfly. Neuroscience 119: 1103-1112, 2003.

Wicklein $\mathbf{M}$ and Varju D. Visual system of the European hummingbird hawkmoth Macroglossum stellatarum (Sphingidae, Lepidoptera): motionsensitive interneurons of the lobula plate. J Comp Neurol 408: 272-282, 1999.

Wylie DR, Bischof WF, and Frost BJ. Common reference frame for neural coding of translational and rotational optic flow. Nature 392: 278-282, 1998

Wylie DR and Frost BJ. Responses of pigeon vestibulocerebellar neurons to optokinetic stimulation. II. The 3-dimensional reference frame of rotation neurons in the flocculus. J Neurophysiol 70: 2647-2659, 1993.

Wylie DR and Frost BJ. Complex spike activity of Purkinje cells in the ventral uvula and nodulus of pigeons in response to translational optic flow. J Neurophysiol 81: 256-266, 1999a.

Wylie DR and Frost BJ. Responses of neurons in the nucleus of the basal optic root to translational and rotational flowfields. J Neurophysiol 81: 267-276, 1999b. 